\title{
Blast Injuries
}

\author{
Wg Cdr M Kumar*
}

\begin{abstract}
Blasts injuries are responsible for about two-thirds of the conventional combat injuries during any military operation and account for a sizeable number during terrorist activities. The pathophysiology, clinical presentation and urgency of management vary significantly in primary blast injuries. These can lead to a wide range of overt and covert injuries, making diagnosis and management difficult. The clinical picture varies from acute respiratory distress to deafness with absence of any external injuries. Such patients are best identified and dealt with by a team of health care professionals well acquainted with battle field injuries.
\end{abstract}

MJAFI 2010; 66 : 309-311

Key Words : Primary blast injury; Blast wave; Barotrauma; Blast lung

\section{Introduction}

$\mathrm{B}$ last injuries have the unparalleled capability to cause several categories of injuries extending from thermal to blunt or penetrating multi-system injuries that are life threatening. These injuries are no longer the forte of the military surgeon what with terrorism reaching the civilian population centres with devastating effects and larger number of casualties among the non-battle ready population.

\section{Historical perspective}

Blast injury was first described in First World War and was thought to involve only the lung. Early literature describes a condition in which soldiers coming in contact with powerful explosions were rendered unconscious or dazed and deaf when no external injury was noted [1]. Knowledge of blast injuries expanded during the Korean and Vietnam wars. About 75 percent of all allied casualties of the first Gulf war [2] and 68 percent of Indian casualties in the IPKF operations were a consequence of blast injuries. A recent study found that 47 percent of blast-related injuries in Iraq involved the head [3].

\section{Mechanism of Blast Injuries}

The nature of injuries caused by an explosion is best understood by analyzing the biomechanics of the blast.

High-energy explosive detonation results in extremely rapid $(0.001 \mathrm{sec})$ conversion of a solid or liquid explosive into gases and sudden changes in atmospheric pressure. The gases expand rapidly, compressing the surrounding air, creating a supersonic "blast over-pressure wave".
Blast strength is described as ratio of the overpressure generated to the ambient pressure. As this wave expands in all directions and the pressure drops, it leads to the creation of the blast "under-pressure wave" with negative pressure and blast winds moving into this area. The resulting extreme pressure differences result in stress and shear forces acting on the susceptible tissues of the human body [4] and are referred to as primary blast injuries. Organs are damaged by dynamic pressure changes at tissue-density (e.g. air-fluid) interfaces due to the interaction of a high-frequency stress wave and a lower-frequency shear wave [5].

The nature of primary blast injuries depends upon the tissue characteristics that are exposed to the blast phenomenon. The advancing edge of the blast wave is called the "blast front". When the blast front reaches the human body it creates an enormous positive pressure shift which is responsible for the tissue damage. The duration of the blast wave is directly proportional to the tissue damage. The blast wave travels at the speed of sound and is more damaging in a medium with reduced acoustic impedance such as underwater blasts and also in closed environs. Added to this is the fact that effects of the blast wave are not prevented by body armour worn by the soldier.

The immediate negative phase of the high pressure blast wave (blast wind) too can cause primary blast injuries by the stress and shear waves produced.

\section{Classification of Blast Injuries}

(a) Primary Blast Injuries (PBI): Injuries caused by the over-pressure blast wave. Almost always affects

*Reader, Department of Surgery, Armed Forces Medical College, Pune-40.

E-mail : drmanojsurgeon@yahoo.co.in 
air-filled structures and structures with air-fluid interface like lung, gastrointestinal tract, tympanic membrane, blood vessels. These are more manifest with high energy explosives.

Tympanic PBI are the commonest and include tympanic membrane rupture and ossicle fracture or dislocation though infrequently hemotympanum without perforation is also seen.

Pulmonary PBI is the cause of mortality in most and includes lung contusion, alveolar rupture, air embolism and ARDS like picture. Air embolism involves cerebral or spinal vessels and produces neurological features indistinguishable from direct injuries.

Cardiovascular changes of PBI include a fall in stroke volume, bradycardia and hypotension.

PBI of the gastrointestinal tract is almost always seen with lung and ear injuries. Intestinal PBI is more commonly seen in underwater injuries and generally involves the fixed parts of the colon with perforation.

(b) Secondary Blast Injuries: Injuries caused by flying shrapnel and present as penetrating organ injury. These are common to any type of explosions and account for the largest class of injuries.

(c) Tertiary Blast Injuries: These are injuries caused due to the blast wind movement moving bodies and debris. Paediatric injuries are more severe due to their smaller body weight and structure [6].

(d) Quaternary Injuries refer to the burns, injuries caused due to toxic fumes, crush injuries, exacerbation of chronic diseases like asthma and psychiatric disorders. These injuries may add to the delayed fatalities.

(e) Quinary Blast Injuries are a more recent addition and occurs commonly after attack with incendiary devices and is characterized by a constant hyperinflammatory state syndrome. This hyperinflammatory state cannot be explained by injuries that are seen after other blast injuries [7]. Present hypothesis is that this state is due to the toxic substances in the blast explosive being inhaled or absorbed through skin. Penta-erythritol-tetra-nitrate (PETN) has been implicated as one such toxin.

\section{Management}

\section{Prehospital Care}

Analysis of blast incidents have shown that reverse triage or "upside-down triage" is common indicating that the less injured access trauma centres earlier than the more severely injured and trapped patients. This can be corrected at the triage stage by evacuating the walkingwounded first.

The healthcare worker responsible for triage should keep in mind that patients with PBI may not have external injuries and may be deaf due to tympanic membrane injuries. Pulmonary symptomatology needs to be given due importance and fluid administration has to be judicious.

Any patient suspected to have been close to the blast area or in an enclosed area should be given high-flow oxygen during evacuation and stabilisation. This is true for all patients with pulmonary symptoms, suspected thoracic trauma and inhalation injuries. Patients with long-bone fractures should be splinted prior to transfer.

\section{Hospital Management}

All patients evacuated from an area close to the explosion should be examined for effects of PBI lung, ear and abdomen even in the absence of other external injuries.

Shrapnel injuries; crush injuries and burns are treated as per standard surgical protocols.

Tympanic membrane Injuries: The $\mathrm{TM}$ is considered more vulnerable than the lung tissue for effects of the blast wave. It is generally accepted that human tympanic membranes begin to rupture at pressures as low as $5 \mathrm{psi}(35 \mathrm{kPa})$ and that majority at 15 psi $(104 \mathrm{kPa})$ [8]. Thus it has traditionally been believed that in the absence of pulmonary symptomatology if the TM is intact, it is highly unlikely that the patient has pulmonary injury. However a recent study of 647 survivors has contradicted this belief [9]. A close correlation to PBI lung or intestinal PBI was not seen.

Over $80 \%$ of tympanic membrane perforations caused by blast are likely to heal spontaneously. Large perforations ( $>80 \%$ surface area) are less likely to heal without surgery [10].

Pulmonary PBI ("Blast Lung") would present with symptoms of dyspnoea, cough (dry to productive with frothy sputum) haemoptysis, and chest pain or discomfort (typically retrosternal). Clinical findings will include tachypnoea, cyanosis, reduced breath sounds and dullness to percussion, coarse crepitations, rhonchi features of pneumothorax or haemo-pneumothorax, subcutaneous emphysema and retrosternal crunch (pneumomediastinum). Patients with blast lung are at risk of pulmonary barotrauma and if there is evidence of tension pneumothorax, it should be decompressed immediately. All patients should receive oxygen at high flow rate (9-15 litres per minute) and injudicious fluid administration avoided.

A chest radiograph is mandatory in all patients exposed to blast which may indicate diffuse infiltrates within the first few hours, progress till 24-48 hours and 
subside within seven days. A delayed picture of the same occurring after 48 hours may be due to complications of blast lung such as ARDS or pneumonia.

Severity of the blast lung may require positive-pressure ventilation with high positive end-expiratory pressure, though with the attendant risk of air-embolism. Various strategies to reduce effects of lung barotrauma include reversion to spontaneous breathing as soon as possible with intermittent mechanical ventilation and CPAP [13]. High frequency ventilation with low airway pressures is recommended for patients with suspected bronchopleural fistula [12].

Blast lung patients are similarly at risk during evacuation by air in partially pressurized military aircraft to larger centres.

Acute Gas Embolism: may be seen clinically as fundoscopy findings, cardiac arrhythmias and CNS or cardiac ischemia. Pulmonary tissue disruptions cause air to move into the arterial system. Management is largely symptomatic and supportive with 100 percent oxygen inhalation. Hyperbaric oxygen therapy has been shown to be effective in patients with early signs of neurological symptoms even when started after 20- 24 hours in stable patients [13].

Abdominal and Pelvic PBI: Air and fluid containing structures are particularly prone for blast injuries with the fixed parts of the colon and mesentery being at the highest risk of perforation and bleeding. Subcapsular haematomas of the solid organs, lacerations, retroperitoneal haematomas, mesenteric ischemia and testicular ruptures have also been described. Patients present with abdominal pain, rectal pain and tenesmus and features of ileus at later stage. Suspicion of bleeding and perforation warrants early exploration. Small bowel contusions $>15 \mathrm{~mm}$ diameter and colonic contusions $>$ $20 \mathrm{~mm}$ diameter at exploration are at higher risk and may need segmental resection. Smaller contusions have lesser chances of late bleed. Focused abdominal sonography in trauma (FAST) has limited role in the detection of bowel or mesenteric injuries but can be useful for detecting fluid collections and some solid organ injuries. Recent studies have supported supplemental imaging using computed tomography and angiography when feasible in civilian set up with limited casualties [14].

\section{Mortality Pattern}

A classical tri-phasic distribution of mortality is seen in blast injuries with high immediate mortality rate owing to complete disruptive injuries, comparatively low rate of early mortality after initial intervention, and a late mortality rate in the hospitalized group of patients [15].

\section{Conclusion}

Multidimensional injury pattern is to be expected in any patient exposed to a blast injury. Key points to be imbibed are that early presentation is the rule with blast lung, abdominal barotrauma may be clinically silent until complications supervene and isolated tympanic membrane barotrauma may not always be a marker for other systemic injuries. Early assessment and prompt management of primary blast injuries will help reduce both early and delayed fatalities.

\section{Conflicts of Interest}

None identified.

\section{References}

1. Mott FW. The effects of high explosives upon the central nervous system. Lancet 1916; 1: 441-9.

2. Spalding TJW. Penetrating missile injuries in Gulf war. Brit J Surg 1991; 78: 102-10.

3. Murray CK, Reynolds JC, Schroeder JM, Harrison MB, Evans OM, Hospenthal DR. Spectrum of care provided at an echelon II medical unit during Operation Iraqi Freedom. Mil Med 2005; 170: 516-20.

4. Kocsis JD, Tessler A. Journal of rehabilitation research and development 2009; 46: 672-7.

5. Guy RJ, Glover MA, Cripps NP. The pathophysiology of primary blast injury and its implications for treatment. Part I: The Thorax. J R Nav Med Serv 1998; 84: 79-86.

6. Quintana DA, Parker JR, Jordan FB. The spectrum of paediatric injuries after a bomb blast. J Paediatric Surg Feb 1997; 32: 307 10.

7. Kluger Y, Peleg K, Daniel-Aharonson L, Mayo A. The special injury pattern in terrorist bombings. J American College of Surgeons 2004; 199: 875-9.

8. Horrocks CL. Blast injuries: Biophysics, pathophysiology and management principles. JR Army Med Corps 2001; 147: 2840.

9. Leibovici D, Gofrit ON, Shapira SC. Eardrum perforation in explosion survivors: is it a marker of pulmonary blast injury? Annals of Emergency Medicine 34: 168-72.

10. Kerr AG. Blast injury to the ear: a review. Rev Environ Health 1987; 7: 65-79

11. Uretzky G, Cotev $\mathrm{S}$. The use of continuous positive airway pressure in blast injury of the chest. Crit Care Med 1980; 8: 486-9.

12. Wanek S, Mayberry JC. Blunt thoracic trauma: flail chest, pulmonary contusion and blast injury. Crit Care Clin 2004; 20 : $171-81$

13. Dunbar EM, Fox R, Watson B, Akrill P. Successful late treatment of venous air embolism with hyperbaric oxygen. Postgrad Med J 1990; 66: 469-70.

14. Sosna J, Sella T, Shaham D, Shapira SC, Rivkind A, Bloom AI et al. Facing the new threats of terrorism: radiologists' perspectives based on experience in Israel. Radiology October 2005; 237: 28-36.

15. Asensio JA, Trunkey DD. Blast Injuries. In: Asensio JA, Trunkey DD, ed. Current therapy of trauma and surgical critical care, First edn. Philadelphia.;Mosby Elsevier 2008; 73-7. 